and $m / n$ lies between constants which depend on $\delta$. Then it is quite easy to prove that

$$
\left|\sigma_{n}^{*}(\theta)\right|=\left|n \sigma_{n}(\theta)-m \sigma_{m}(\theta)\right| /(n-m) \leqq M B,
$$

while your argument shows at once that $\left|R_{n}(\theta)\right| \leqq 2 B+\vartheta A$, where $\vartheta$ is small by choice of $m / n \ldots . " \dagger$

UNIVERSITY OF BUDAPEST

\title{
NOTE ON THE FORM OF A FIRST-ORDER PARTIAL DIFFERENTIAL EQUATION
}

BY A. B. BROWN

In this paper we give a simple proof of the fact that the non-singular solutions of a first-order partial differential equation can be obtained by equating to zero solutions of an associated equation in which the dependent variable does not appear explicitly. The usual proof $\S$ of this property makes extensive use of the complete integral, and to be given rigorously would require considerations at one stage nearly as involved as our entire proof.|| Our proof has no reference to complete integrals. The results, as usual, hold in the small. Interest in this question arises from the treatments of equations in which the unknown does not appear explicitly.

TheOREM. Let $f\left(x_{1}, \cdots, x_{n}, z, p_{1}, \cdots, p_{n}\right)=f(x, z, p)$ be of class $C^{\prime \prime} \mathbb{T}$ in a neighborhood of an initial element $\left(a, b, p^{0}\right)$ for which $f=0$ and $f_{p_{1}} \neq 0$. Let

$\dagger$ Professor Fekete, to whom I communicated this letter of Paley in September 1933, has worked out completely the proof sketched by Paley. Moreover, Fekete generalized considerably Paley's theorem and extended it also to the trigonometric series of $\mathrm{H}$. Bohr.

$\ddagger$ Presented to the Society, March 31, 1934.

$\S$ See, for example, E. Goursat, Équations aux Dérivées Partielles, 1921, pp. 48-49 and 159 .

\| A complete integral yielding elements at a given point does not necessarily provide any given integral element at the point.

If A function of class $C^{(k)}$ is one having continuous $k$ th partial derivatives. 


$$
\begin{aligned}
& F\left(x_{1}, \cdots, x_{n}, z, P_{1}, \cdots, P_{n+1}\right) \\
& \quad \equiv f\left(x_{1}, \cdots, x_{n}, z,-\frac{P_{1}}{P_{n+1}}, \cdots,-\frac{P_{n}}{P_{n+1}}\right) .
\end{aligned}
$$

Then the solutions of class $C^{\prime}$, with elements near $\left(a, b, p^{0}\right)$, of

$$
f(x, z, p)=0, \text { with } \frac{\partial z}{\partial x_{k}}=p_{k}, \quad(k=1, \cdots, n),
$$

are identical with the loci expressible in the form

$$
g\left(x_{1}, \cdots, x_{n}, z\right)=0
$$

for which

$$
u=g\left(x_{1}, \cdots, x_{n}, z\right), \quad g \text { of class } C^{\prime}, \quad g_{z} \neq 0,
$$

is a solution of

$$
F(x, z, P)=0, \quad \text { with } \quad P_{k}=\frac{\partial u}{\partial x_{k}}, \quad(k=1, \cdots, n),
$$

$$
P_{n+1}=\frac{\partial u}{\partial z} \neq 0
$$

and with $\left(x_{1}, \cdots, x_{n}, z,-P_{1} / P_{n+1}, \cdots,-P_{n} / P_{n+1}\right)$ near $\left(a, b, p^{0}\right)$.

It is easily verified that if (4) satisfies (5), then (3) yields a solution of (2). Conversely, if (3), with $g_{z} \neq 0$, satisfies (2), then (4) satisfies (5) when $g=0$, but not necessarily elsewhere.

Let a solution of (2) be given in the form

$$
z=Z\left(x_{1}, \cdots, x_{n}\right), \quad Z \text { of class } C^{\prime} .
$$

By a change in notation we can assume that $b=Z\left(a_{1}, \cdots, a_{n}\right)$, and that $\partial Z\left(a_{1}, \cdots, a_{n}\right) / \partial x_{k}=p_{k}{ }^{0},(k=1, \cdots, n)$. Taking $g(x, z)=Z(x)-z$, the concluding sentence of the preceding paragraph shows us that (7) is an integral element of (5):

$$
\begin{aligned}
& \left(x, z, u, P_{1}, \cdots, P_{n}, P_{n+1}\right) \\
& \quad=\left(a, b, 0, Z_{x_{1}}(a, b), \cdots, Z_{x_{n}}(a, b),-1\right) .
\end{aligned}
$$

Note that $P_{n+1}=-1 \neq 0$, for this element.

Since $F_{P_{1}}=-f_{p_{1}} / P_{n+1} \neq 0$ for ( 7$)$, the manifold 


$$
x_{1}=a_{1}, \quad u=Z\left(a_{1}, x_{2}, \cdots, x_{n}\right)-z,
$$

with the integral element (7), determines uniquely an integral strip for (5), which in turn determines uniquely a solution of the equation $(5): *$

$$
u=G\left(x_{1}, \cdots, x_{n}, z\right),
$$

where $G$ is of class $C^{\prime}$, with

$$
G\left(a_{1}, x_{2}, \cdots, x_{n}, z\right)=Z\left(a_{1}, x_{2}, \cdots, x_{n}\right)-z .
$$

We now consider the locus

$$
G(x, z)=0
$$

near $(a, b)$. Since $G_{z}(a, b)=-1 \neq 0$, we infer that (10) determines a locus which we know satisfies (2). Now the locus

$$
x_{1}=a_{1}, \quad z=Z\left(a_{1}, x_{2}, \cdots, x_{n}\right),
$$

with $p_{k}=Z_{x_{k}},(k=1, \cdots, n)$, yields an integral strip, say $S_{1}$, for (2), since (6) satisfies (2). But (11), with $\partial z / \partial x_{k}=-G_{x_{k}} / G_{z}$, also yields an integral strip, say $S_{2}$, for (2); for (9) shows that (11) is the part of the locus (10), obtained by setting $x_{1}=a_{1}$, and (10) satisfies (2).

At $(a, b),-G_{x_{k}} / G_{z}=-P_{k} / P_{n+1}=Z_{x_{k}}(a, b),(k=1, \cdots, n)$, as we see from (7). Hence $S_{2}$ has an element in common with $S_{1}$, and as $S_{1}$ and $S_{2}$ both have the locus (11) in $(x, z)$-space, it follows that they are identical. Therefore the solutions of (2) determined by them, namely (6) and (10), are likewise identical. Recalling that (8) satisfies (5) and that $G_{z} \neq 0$, we see that the theorem is established.

It would be interesting to know whether the theorem is true in the large.

Columbia University

* See L. Bieberbach, Differentialgleichungen, 3d ed., 1930, pp. 294 ff. The argument is in the small throughout. 